\title{
Improving Energy Efficient QOS Performance for Heterogeneous MANET
}

\author{
S. VENKATASUBRAMANIAN ${ }^{1}$ and N.P. GOPALAN ${ }^{2}$ \\ ${ }^{1}$ Associate Professor, Saranathan College of Engineering, Department of Computer Science \& \\ Engineering, Anna university, Chennai \\ ${ }^{2}$ Professor, National Institute of Technology, Computer Science \& Engineering, Tiruchirapalli, Tamilnadu \\ E-mail: '
}

\begin{abstract}
In Mobile Ad hoc Networks, routing is one of the most essential tasks owing to volatile topology. In MANET, the nodes can depart and connect the network; this uneven nature makes support of QoS (Quality of Service) is a complex task. A major issue in heterogeneous networks is energy utilization. In this paper, a heterogeneous network is intended to attain enhanced energy-efficient multipath QoS provisioning. Primarily, the multipath routes are assumed to be discovered and they then find the gateway node along with all the nodes in the network by using the gateway selection procedure. By using a QoS based Energy Efficient Optimal Multipath route discovery and route maintenance in multipath routing protocol, discover the optimal path for data transmission from the source to the destination. In this way, QoS parameters such as bandwidth, throughput, packet delivery ratio and end-to-end delay will be computed and accomplish in the best possible way.
\end{abstract}

Keywords: Heterogeneous MANET, multipath routing, gateway selection, gateway migration and QoS provisioning.

\section{INTRODUCTION}

The Mobile Ad Hoc Networks (MANETs) are highly dynamic in nature and infrastructure free. It is consisted of a group of mobile nodes which are not surrounded by any infrastructure. Because of non-restricted mobility these nodes are liberated to move autonomously in any direction and the straightforward deployment's uniqueness of MANETs make them incredibly popular and highly appropriate for applications like emergencies, natural disasters and military operations.

One of the budding researches in recent years is that of Heterogeneous mobile ad-hoc networks (MANETs). A network that links computers and

Supplementary devices with the unrelated operating system and/or protocols is a heterogeneous network. The Heterogeneous Wireless Network (HWN) architecture, which is capable of providing elevated data-rate and end-to-end connectivity makes use of the superior bandwidth of multi-hop networks and the wide-range communication of $3 \mathrm{G}$ Networks as well. Although homogeneous MANETs are easy to design and analyze, they exhibit pitiable flexibility and scalability when compared with heterogeneous MANETs that consist of distinct mobile nodes with distinct resources in the network [1]. The heterogeneous MANETs are the wireless networks which incorporate multiple wireless nodes and make connectivity and data access everywhere possible at any time. It is merely the integration of Mobile Ad hoc Network, Wireless LAN and Cellular networks. The gateway nodes act as liaisons between the wireless networks for connection establishment. A gateway node is the combination of routers and wireless access points which as well as makes available firewall functions for wireless connectivity and its maintenance [2].

Several quantitative and qualitative factors from end-to-end delay, bandwidth and transmission reliability to privacy are depicted as Quality of 
service [3]. MANETs experience a number of difficulties; some of them are inadequate communication range, battery life and also limited knowledge of their environment. To attain a supplementary deterministic network performance is the aim of QoS provisioning. The performance of the network is evaluated based on the Quality of Service; these QoS parameters may differ from one application to another [4].

Energy conservation is one of the most important criteria for optimizing the nodes. Energy efficient routing has a considerable impact on the MANETs due to the restrictions of the mobile node's battery power. These batteries cannot be alternated and recharged in complex scenarios such as battlefields and emergency relief scenarios. For this drawback, the nodes in Ad Hoc networks should be permitted to manage their energy utilization efficiently to prolong the network's lifetime. The energy utilization of every node differs according to its communication state such as transmitting, receiving, listening or sleeping. When the power of a node gets depleted it will affect the entire network's lifetime [5]. Saving energy for mobile nodes is one more serious problem because if a node's battery is exhausted it cannot function at all. Node breakdown can also cause network separation which will lead to the entire network's failure and no service provisioning as well. Hence, energyefficient and routing protocols have to get a great deal of research concentration [3]. As a result, energy efficiency should be taken into consideration as it is a critical and extensive research issue.

In this paper, a method which provides the architecture for energy-efficient optimal multi-path routing in the heterogeneous MANET is proposed. Total energy consumption is divided into two parts, path-discovery and packet-transmission. Based on metrics like mobility speed and the remaining energy of the node, a gateway is elected. The selected gateway must be able to enhance connectivity to provide a service for a longer time. So, the Gateway node which is elected as the current gateway should have higher remaining energy. There are multiple routes between the source and the destination in which the route having maximum energy is selected as the optimal route. The data is transmitted through this optimal route and in this way QoS parameters such as bandwidth, throughput, packet delivery ratio and end-to-end delay will be calculated in the best possible manner.

The rest of the article is structured as follows: Section 2 describes some of the related works. Detailed explanation about the proposed work is presented in section 3. Section 4 illustrates the architecture of the proposed method. Finally, in the last section simulation results and conclusions are summarized.

\section{RELATED WORKS}

An Adaptive Gateway Management (AGM) method was projected by Manoharan et.al [2], which has to pick the most excellent gateway from the gateway candidate nodes. Based on the metrics like remaining energy, $3 \mathrm{G}$ signal power and mobility speed these nodes were selected. Once an elected gateway loses its optimality owing to diminishing energy and dynamic unstable topology, it makes it unavailable which results in the disconnection between the networks. In order to preserve the connectivity between networks for a longer time the Adaptive Gateway Migration method had also intended to pick an optimal gateway to which the duty of the existing gateway was migrated, only when the above mentioned metric values achieved a predefined threshold.

Soundararajan, S et.al [6], have projected a congestion controlled adaptive multi-path routing protocol to attain load balancing and avoid congestion in MANETs. The algorithm for finding multi-path routes calculates fail-safe multiple paths, which then provide all the intermediate nodes on the primary path with multiple routes to the destination. The nodes with the smallest amount of load, additional battery power and remaining energy are integrated in the fail-safe multipath. When the average load of a node along the route increases beyond a threshold, it distributes the traffic over disjoint multi-path routes in order to reduce the traffic load on a congested link.

For link constancy and least quantity of drain rates energy utilization, Florin De Rango et.al [7] have projected an approach for routing. Link stability and Energy aware Routing protocol (LAER) were proposed and a biobjective optimization formulation was designed to validate the exactness of the projected resolution. The protocol routine had been estimated in terms of Data Packet Delivery Ratio, Normalized Control Overhead, Link duration, Node's lifetime and Average energy consumption.

Chandrakant $\mathrm{N}$ et.al [8], have projected a set of heterogeneous nodes in MANETs which were having different packet sizes, uncommon protection mechanisms, uncommon communication speeds, etc. The structural design they projected encompasses two main components, security management and communication management. In this network every node did not understand security 
methods of every other node because there could be diverse encryption techniques, packet sizes, protocols, etc. This method was very important for the protection issues in the heterogeneous nodes in MANETs.

\section{SYSTEM DESIGN AND PROTOCOL}

Networks that link computers and supplementary devices with an unrelated operating system and/or protocol are heterogeneous networks. For example, a wireless network which gives a service through a wireless LAN and can retain the service when switching to a cellular network is called a wireless heterogeneous network. The major intention of the heterogeneous MANET is to incorporate different wireless network models namely Cellular network, WLAN and $3 \mathrm{G}$ which give seamless connectivity and end-to-end data transfer between dissimilar networks working under dissimilar environments [2]. So by transferring data from one MANET to another QoS is unachievable but by electing the appropriate gateway we can achieve these QoS. This is possible because it can act as a bridge between one MANET and another i.e. it will convert one protocol into another. Energy utilization in a heterogeneous network is a vital problem depending on whether they function in a free-standing Mobile Ad Hoc Network (MANET). The life span of a network will be enhanced by suitably reducing the necessity of power for connections. There is a challenge to provide Quality of Service (QoS) clarifications to wireless domains and retain the QoS in an ad hoc network [24]. Our proposed approach requires an energyefficient optimal multipath which will provide all the intermediate nodes on the primary path with multiple routes to the destination. There is a need to discover multiple paths for a given source and destination; and the best paths should be selected for transmission.

\subsection{Qos Based Energy Efficient Optimal Multipath Routing Protocol}

Initially, the multipath routes are assumed to be discovered by broadcasting an RREQ message from the source to a node on the path. If there is a path available, send an RREP message and this process will continue until it reaches the destination. An Ad-hoc On-demand Multipath Distance Vector Routing (AOMDV) protocol is used for computing numerous loop-free and link disjoint paths [10]. In the AOMDV every route request and every corresponding route reply incoming at a node potentially defines an exchange path to the node. The "advertised hop-count" is introduced to remove any opportunity of loops. The protocol accepts only the swap routes with hopcount lesser than the advertised hop-count and swap routes with higher or the equivalent hop-counts are discarded. The advertised hop-count method launches multiple loop-free paths from each node and these paths have be disjoint. When a node sends a route request packet in the network every node will receive the route request incoming at an intermediate node through a dissimilar neighbor of the sending node or the node itself. This is used inbetween nodes in the AOMDV protocol for eliminating the duplicate copies of the route requests. Each and every packet is checked to observe if it supplies a node-disjoint path to the source. This is confirmed with the initial hop field in the route request packet and the initial hop list for the route request packets at the node. At the target in the paths determined there is a link disjoint and not a node disjoint. The target replies with $\mathrm{k}$ copies of the route request by not considering the first hops. The route requests need to reach the destination through unique neighbors. The multiple routes are discovered for each node in the network. After discovering multiple paths for a given source and destination, the optimal path should be selected for transmission.

When a node enlarges the communication, the power utilization will also increase because a node consumes power from its battery for every transmission and reception of data packets. Because MANETs have limited energy resources for communication, usage of the energy resources of a small set of nodes at the cost of others can have an adverse impact on the nodes' lifetime as well as network's lifetime [5]. Each and every node in the network is driven by a battery and it has only a limited energy supply. Most of the protocols have been designed to ensure longer battery life. The nodes in the network are powered even though they are not performing any useful work. By overhearing other transmissions in the network the useful energy of the nodes is wasted. Based on decreasing the hop count or delay, the paths are computed in routing protocols. Thus, a number of nodes become involved in routing packets for numerous source-destination pairs. The energy resources of these nodes get depleted quicker than other nodes, which in some cases may lead to separation of the network, thus decreasing the lifetime of the network. To identify the availability of the paths from source to destination, broadcast the RTS message from the source. The consumed energy for transmitting RTS from source to destination is given by: 


$$
\text { Energy }^{R T S}=\rho * t_{N}^{L} * p_{R T S}
$$

The consumed energy for transmitting CTS from destination to source is give by

$$
\text { Energy }^{C T S}=\rho * t_{N}^{L} * p_{C T S}
$$

Where, $\rho$ is the receiver's threshold, $t_{N}$ is the maximum transmission range, $L$ is the path loss exponent and $p_{R T S} \& p_{C T S}$ is the transmission time of RTS and CTS. The source that calculates the total energy consumption of each path while transmission of RTS and CTS is given as

$$
\text { TotalEnergy }^{E}=\text { Energy }_{j}^{R T S}+\text { Energy }_{j}^{C T S}
$$

Based on the battery power consumed at each node in the route, the residual battery power over each route is calculated as

$$
R_{i}^{b}=\sum_{i \in M_{L}} R_{i}
$$

Where $M_{L}$ is the number of nodes in the chosen path. The multipath which has maximum energy is selected as an optimal path. By using this optimized route, the data is transferred from the source to destination from one network to another with the help of a gateway node.

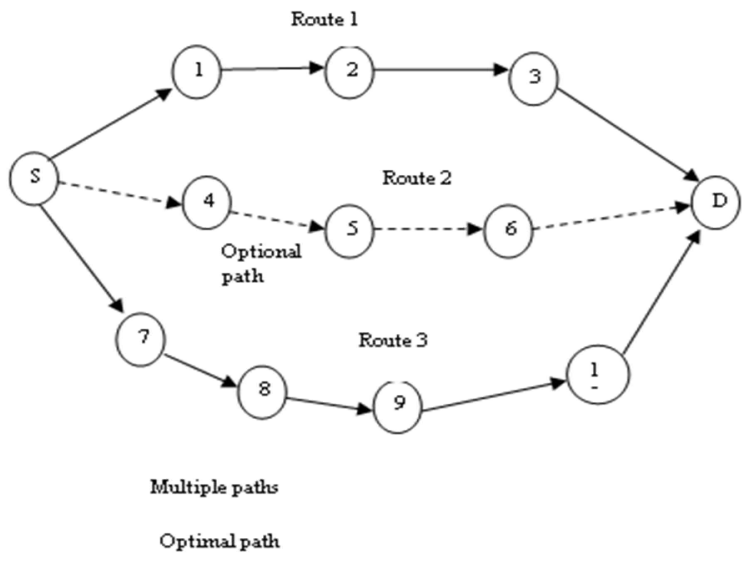

Fig. 1. Optimal path selection

The routes from source to destination are discovered and then the optimal path which is denoted by a dotted line is selected from the already discovered multipaths which are indicated by solid lines.

\subsection{Gateway Selection}

A Gateway node can function in any network layer. It is used to translate the standard protocol of one device to another which is necessary for other devices to accomplish interoperability which also translates the data formats, transmission rates and protocols of one network into the protocols of the network in which data is being navigated. In this section we suggest a novel technique to select the gateway. It is essential to select a gateway with an efficient QoS to get better performance such as network throughput, packet delivery ratio, end to end delay and bandwidth. An internet working system capable of fusing two networks that uses dissimilar protocol is a gateway. The Gateway node acts as a link connecting multiple networks. The gateway discovery and selection is a vital factor to enable the integration between different types of networks [11]. Select a gateway node from each network in the heterogeneous MANET. By comparing other nodes, the gateway node is equipped with a more dominant communication capacity. Data can be obtained from a MANET through the gateway at any time and any place. It performs routing across multiple MANETs; by consuming the advanced capabilities, gateways can connection to any technology. The gateway selection procedure is based on the metrics such as mobility speed, remaining energy and signal strength of the nodes in the MANET. All nodes in the MANET will broadcast a gateway request to each other and the nodes will reply with a hello message along with the metric information. The metric values fall in different ranges and different scales of evaluation. These values are scaled and brought into non-dimensional values. The scale metric value of each node is calculated. The scaled methods with criteria are of two types i.e. positive and negative. Interconnection of networks to last long with high data rate services belongs to the metrics with positive criteria. Metrics with positive criteria comprise of remaining energy and signal strength. For calculating the positive criteria the formula is given as

$\delta=\frac{Y-Y_{\text {min }}}{Y_{\text {max }}-Y_{\text {min }}}$

Mobility metrics with negative criteria which is for rising mobility lead to routing overhead and congestion which in turn reduces throughput and performance of the network. For calculating the negative criteria the formula is given as 
$\delta=\frac{Y_{\max }-Y}{Y_{\max }-Y_{\min }}$

Where, $Y$ is the value of each metric and $Y_{\min }, Y_{\max }$ is the maximum and minimum metrics considered among all the nodes and then calculate the weight of each node. The sum of weight is always equal to one. The total additive weight of nodes is calculated as

$$
W_{\max }=\sum_{j=0}^{m}\left(\text { Weightfometitymetri }^{*} \text { scalemetrivalu } \phi\right.
$$

Where, $m$ is the total number of metrics. The node which has maximum weight is considered as a gateway. After the gateway is selected, it broadcasts a hello message with the Gateway to all the nodes in the MANET. Activate the interface on the current gateway node. When the nodes are connected in a network, the interface nodes act as an interconnecting network for seamless integration and for end-to-end connectivity.

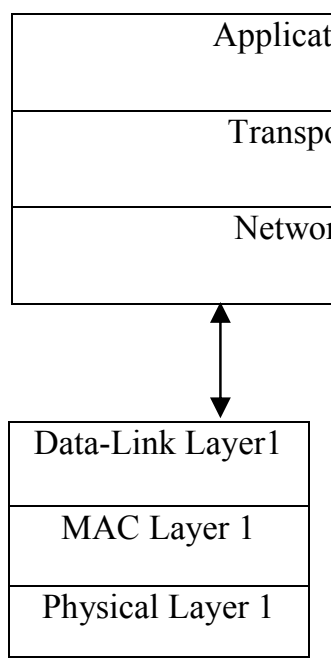

Bluetooth

\section{Wi-Fi}

Fig. 2. Protocol stack of dual interface MS with Bluetooth and Wi-Fi

The protocol stack of a dual-mode Mobile Station which consists of multiple layers is depicted in Fig. 3. The dual interface consists of the Physical layer, Media Access Control (MAC) layer and Data Link Layer which are designed independently. The rest of the layers are used for communicating with each other, which are network layer, Transport layer and Application layer. Through the physical layer, users send the packet to the gateway, following the MAC layer and Data Link Layer investigation, the data can be transferred to the Network layer to be another communication protocol and then the packet is encapsulated in the following order: Data Link Layer, MAC layer and Physical layer and then sent to the target [16]. Choosing the most suitable combination of inferior layers that provide the most excellent service to the superior layers became a critical issue. To run independently, the network layer provides the homogeneous substrate over the transport and application layers.

To provide access for ad hoc nodes, an interface between the ad hoc network and the access network is needed. We use the dual interface that establishes a connection over a heterogeneous network. In order to be able to route packets among these dissimilar networks, the interface needs the protocols and standards of both the networks. The protocol stack of the dual interface is depicted in Figure 3.

\begin{tabular}{|l|l|}
\hline Higher levels & Higher levels \\
\cline { 1 - 1 } IPv4 & IPv6 \\
\cline { 1 - 1 } SNDP/PDCP & $\begin{array}{l}\text { Data Link } \\
\text { Layer }\end{array}$ \\
\cline { 1 - 1 } LLC/PDCP & Physical layer \\
\cline { 1 - 1 } RLC & \\
\hline MAC & \\
\hline
\end{tabular}

Fig. 3. Protocol stack of dual interface $M S$ with $3 G$ and $\mathrm{Wi}-\mathrm{Fi}$

WLAN makes use of physical and data link layers of the IEEE 802.11 standard. On the $3 \mathrm{G}$ network side, the interface uses standard UMTS physical and data link layer protocols. To recognize two different protocol stacks on the dual interface it needs two different network interfaces.

\subsection{Selection Of New Gateway}

The most vital issue in MANET is the dynamic changing topology. The topology alterations are characterized as the nodes belonging to a particular MANET can become separated into numerous subMANETs owing to node mobility. The gateway should notice the topology variations in every subMANET. If the essential routing protocol of the MANET is proactive, the separation will be noticed automatically by the fundamental routing protocol. However, if a separation happens in a reactive MANET, the happening may not be noticed for a while awaiting a new significance which needs to 
be sent to a destination in a disconnected partition [10]. A single node breakdown in networks is typically unimportant if it does not lead to a defeat of sensing and communication coverage. MANETs are oriented towards personal communications and the defeat of connectivity to any node is important. Thus, energy efficient routing is one of the supreme significances of a MANET's design. When the gateway node moves through the network, the range of the gateway node gets depleted and then the network will select a new gateway, otherwise when the energy of the gateway node gets depleted a new gateway is selected from the nodes present in the network. After selecting a new gateway node, the current gateway will forward all the incoming connections to the new gateway and deactivates all the interfaces from the current gateway.

\section{ARCHITECTURE}

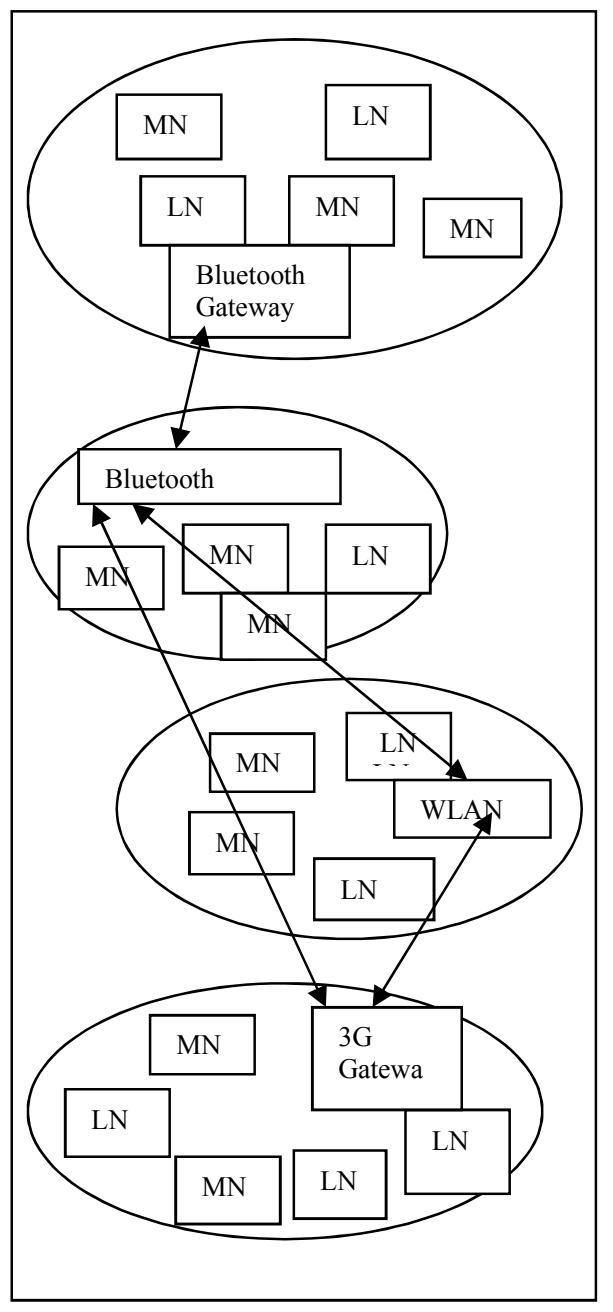

MN-Mobile node

LN - Laptop node

Fig. 4. Heterogeneous MANET
The structure of a Heterogeneous MANET is depicted in figure 4. It consists of a WLAN, a $3 \mathrm{G}$ and WLAN network and a $3 \mathrm{G}$ and Bluetooth network. In the Bluetooth network, the packets are exchanged through frames. These frames are transmitted on a dissimilar hop frequency. Asynchronous data channels are maintained by the Bluetooth. The general packet format for Bluetooth is depicted in figure 5. These frame fields have access codes; the Header and the payload. The access codes which are for identity are Channel access codes (CAC) to classify a piconet, Device access codes (DAC) used for paging and subsequent responses and Inquiry access codes (IAC) used for investigation functions.

\begin{tabular}{l|l|l|}
\hline $\begin{array}{c}\text { Access } \\
\text { Code }\end{array}$ & Header & Payload \\
\hline 72 bit $\quad 54$ bit $\quad 0-2745$
\end{tabular}

Fig. 5. Packet format for Bluetooth

The Wi-Fi network uses IEEE 802.11 standard. Duration, CFP frames and PS-Poll frames are the three forms of the duration ID field. Depending on the frame type, different fields are used for different purposes and these address fields are numbered. The sequence control field is used for both defragmentation and also removing duplicate frames. The Frame Check Sequence permits the stations to verify the integrity of the received frames.

After constructing the network, by using routing protocol discover multiple routes for the nodes in the network. Then by using a gateway selection algorithm select a gateway node. A Gateway node is selected for each network. From the multiple paths pick an optimal path; send the packet through the selected optimal path from the source to the destination. Nodes in MANET are battery powered. Energy conservation is one of the most important criteria for optimizing these nodes. The optimal path is selected based on the maximum residual energy in the path. While sending the packet, firstly it is forwarded to the gateway node of that particular network. That gateway node will send the data packet to that network gateway where the destination is present. Then from that gateway node the data is sent to the destination. The use of gateway nodes is to convert the packet format based on the network and send the packet from one network to another. If the gateway nodes get depleted, then the network will select a new gateway node. The existing gateway will forward all the incoming connections to the newly selected 
S. Venkatasubramanian et al. / International Journal of Computer Networks and Communications Security, 2 (2), February 2014

gateway and then deactivate the interface. The new gateway will act as a gateway in the network. Once a node of a MANET moves away from its region, the node gets disconnected from the MANET. Suppose the node enters into the region of a neighboring MANET, it can connect with that MANET after authentication. We have simulated nodes with unique properties range, battery power, etc. So it can easily identify the homogeneous node.

\begin{tabular}{|l|l|l|l|l|l|l|l|l|}
\hline $\begin{array}{l}\text { Frame } \\
\text { Control }\end{array}$ & $\begin{array}{l}\text { Duration } \\
\text { ID }\end{array}$ & $\begin{array}{l}\text { Address 1 } \\
\text { (Receiver) }\end{array}$ & $\begin{array}{l}\text { Address 2 } \\
\text { (Sender) }\end{array}$ & $\begin{array}{l}\text { Address 3 } \\
\text { (Filtering) }\end{array}$ & $\begin{array}{l}\text { Sequence } \\
\text { Control }\end{array}$ & $\begin{array}{l}\text { Address 4 } \\
\text { (Optional) }\end{array}$ & Frame Body & FCS \\
2 bytes 2 bytes & 6 bytes & 6 bytes & 6 bytes & 2 bytes & 6 bytes & $\begin{array}{l}0-2132 \\
\text { bytes }\end{array}$ & 4 bytes
\end{tabular}

Figure 6: Packet format for IEEE 802.11

\section{EXPERIMENTAL RESULTS}

The proposed heterogeneous MANET has been implemented in java. In our simulation, we use 50 nodes with a 1000 meter $\times 700$ meter rectangular region for 60 seconds simulation time. Initially each node in the MANET is provided with 20J and is enabled with IEEE 802.11 interface. The data transmission rate is $0.5,1.0,1.5$ and $2 \mathrm{Mb}$. While transmitting and receiving, $0.6 \mathrm{~J}$ and $0.3 \mathrm{~J}$ are reduced from the initial energy. The simulation parameters used in this work is summarized as given below.

Table1: Simulation parameters

\begin{tabular}{|l|l|}
\hline No. of Nodes & 50 \\
\hline Area Size & 1000 X 700 \\
\hline Mac & 802.11 \\
\hline Simulation Time & $60 \mathrm{sec}$ \\
\hline Packet Size & 512 \\
\hline Transmit Power & $0.6 \mathrm{~J}$ \\
\hline Receiving Power & $0.3 \mathrm{~J}$ \\
\hline Initial Energy & $20 \mathrm{~J}$ \\
\hline Transmission Rate & $0.5,1.0,1.5$ and2Mb \\
\hline
\end{tabular}

\subsection{Results and Discussions}

\subsubsection{Performance Metrics Based On Nodes}

The performance is calculated based on the following metrics. In our experiment the results are based on varying the number of nodes as $20,30,40$, 50 and 60.

\subsubsection{Packet Delivery Ratio}

Ratio of the number of packets received effectively and the total number of packets transmitted.

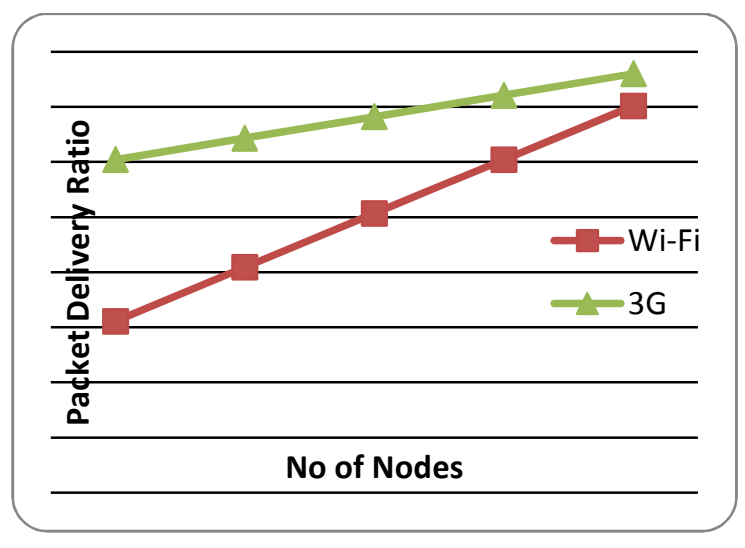

Fig. 7. Comparison of Packet Delivery Ratio between WiFi and $3 G$ 
The Packet Delivery Ratio of $3 \mathrm{G}$ has better performance when compared with Wi-Fi which is depicted in Figure 7. This is because in the time waited at a node, our protocol can find an alternate path if the current link has broken. As the number of nodes increases, the packet delivery ratio will also get increased in both the networks.

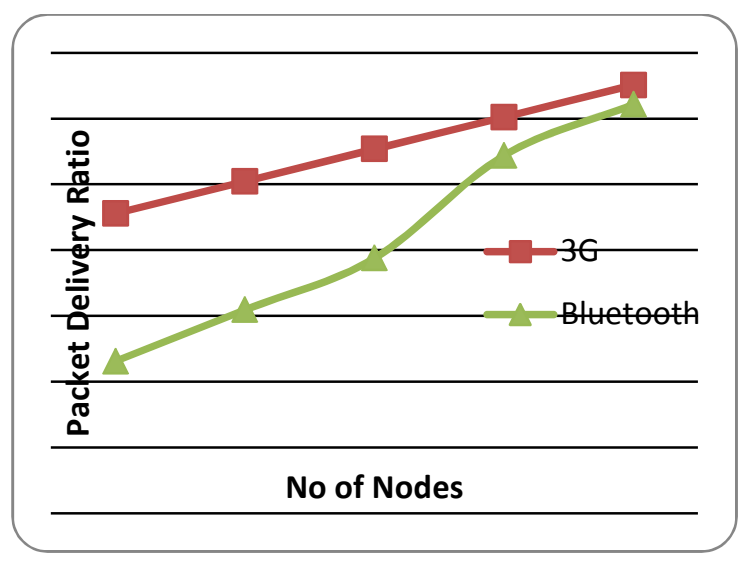

Fig. 8. Comparison of Packet Delivery Ratio between $3 G$ and Bluetooth

In Figure 8, we note that the $3 \mathrm{G}$ network has a better packet delivery ratio when compared to the Wi-Fi network for each set of connections.

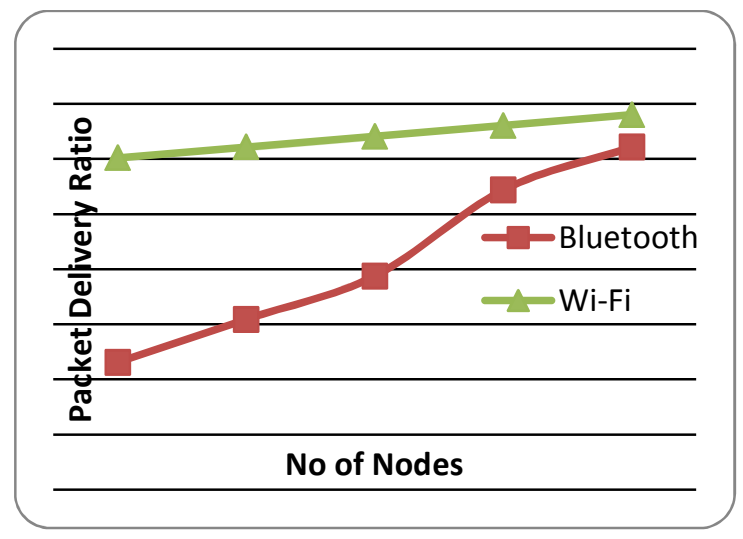

Fig. 9. Comparison of Packet Delivery Ratio between Bluetooth and $\mathrm{Wi}-\mathrm{Fi}$

In Figure 9, the packet delivery ratio of the Wi-Fi network has a better result when compared with the Bluetooth. It can be observed from these figures that the proposed protocol has better packet delivery ratio when the simulation is carried out and it increases because the number of packets are received through the selected gateway. As observed from these figures, the proposed EEQMRP protocol gives better packet delivery ratio for $3 \mathrm{G}$ network when compared with the other networks.

\subsubsection{End-To-End-Delay}

Amount of time taken by the packet to arrive at the destination.

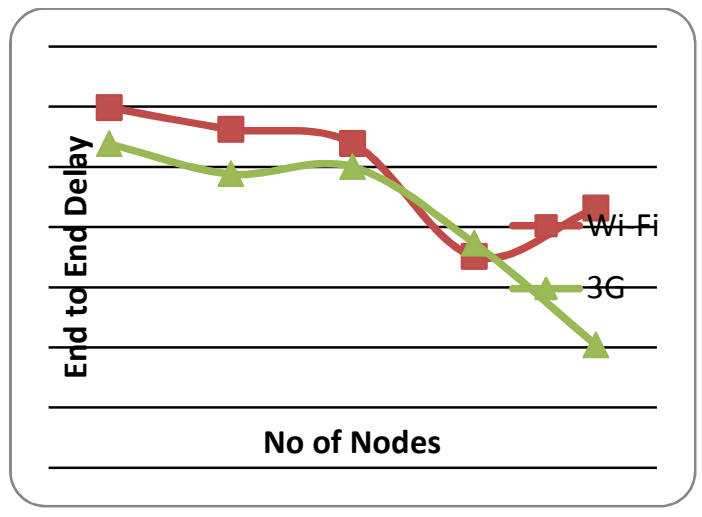

Fig. 10. Comparison of end to end delay between $\mathrm{Wi}-\mathrm{Fi}$ and $3 G$

It indicates the length of time taken for a packet to travel from the source to the destination. It represents the average data delay an application or a user experiences when transmitting data. The comparison between $\mathrm{Wi}-\mathrm{Fi}$ and $3 \mathrm{G}$ for end to end delay is show in Figure 10. It shows that the $3 \mathrm{G}$ network obtained a better result while comparing it with the Wi-Fi network.

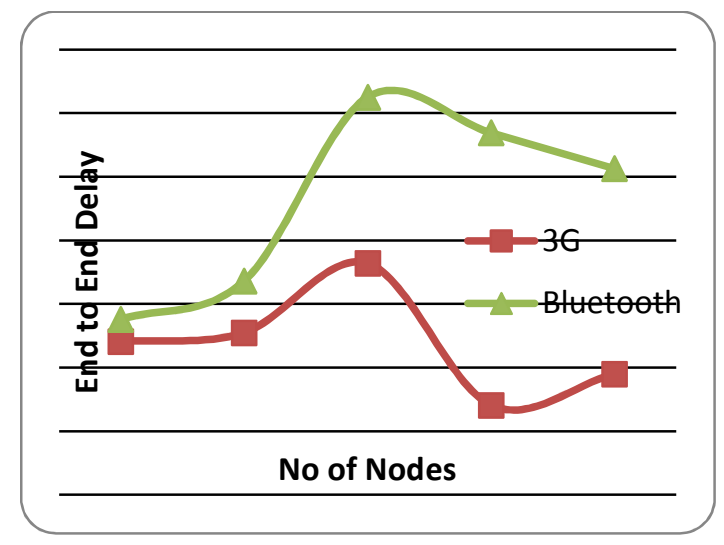

Fig. 11. Comparison of end to end delay between $3 G$ and Bluetooth

In Figure 11, we note that the $3 \mathrm{G}$ network has a better end to end delay when compared with the Bluetooth network for each set of connections. 
S. Venkatasubramanian et al. / International Journal of Computer Networks and Communications Security, 2 (2), February 2014

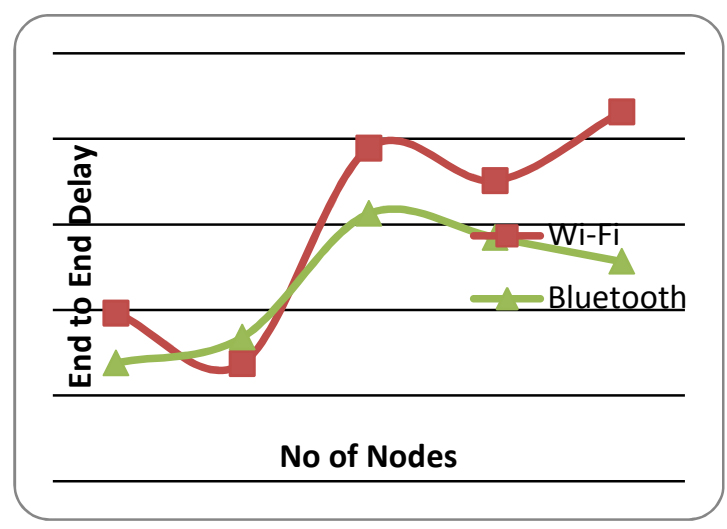

Fig. 12. Comparison of end to end delay between $\mathrm{Wi}-\mathrm{Fi}$ and Bluetooth

In Figure 12, we note that the $3 \mathrm{G}$ network has a better packet delivery ratio when compared to the Wi-Fi network for each set of connections. From these results we conclude that our proposed protocol has a better end to end delay when the simulation is carried out and it increases because the number of packets is received through a selected gateway. As observed from these figures the proposed EEQMRP protocol gives better end to end delay for the $3 \mathrm{G}$ network when compared with the other networks.

\subsubsection{Bandwidth}

The available bandwidth based on the channel status of the network.

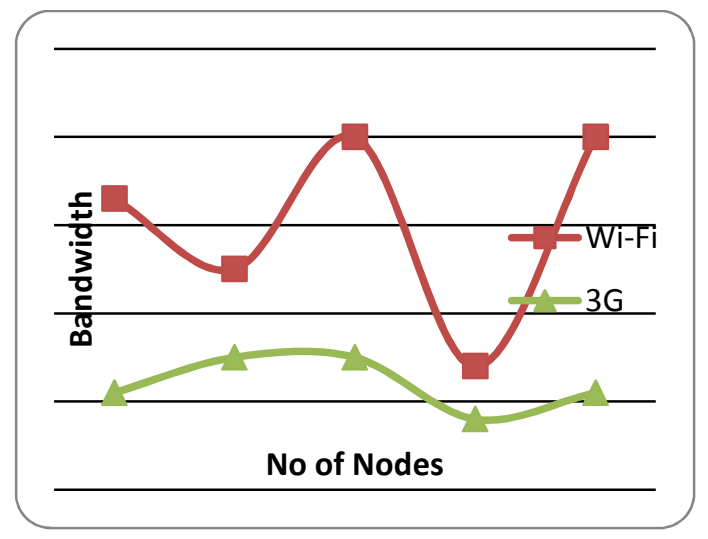

Fig. 13. Comparison of bandwidth between Wi-Fi and $3 G$

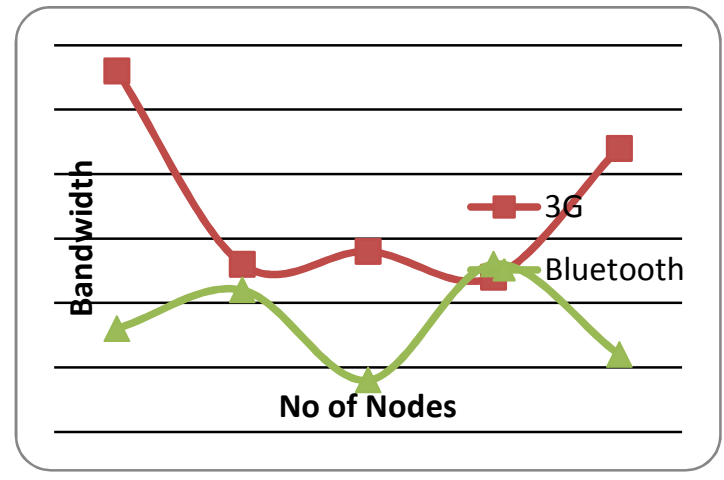

Fig. 14. Comparison of bandwidth between $3 G$ and Bluetooth

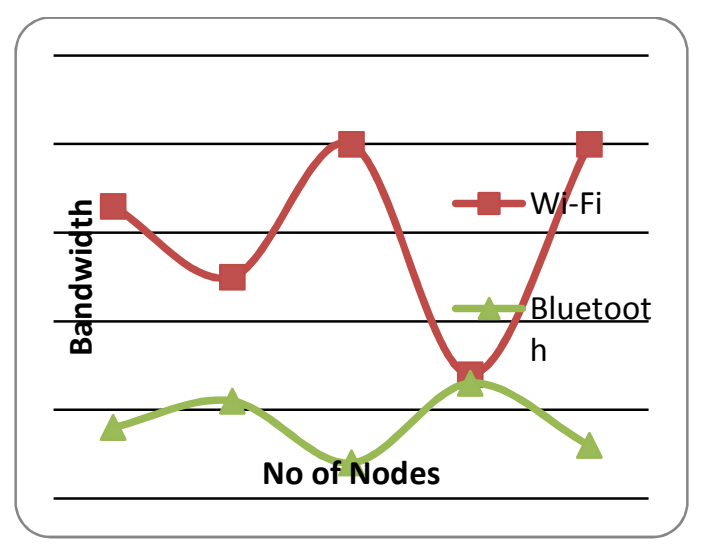

Fig. 15. Comparison of bandwidth between Wi-Fi and Bluetooth

\subsubsection{Energy Consumption}

It is calculated from the primary energy and the remaining energy of all nodes associated with the network.

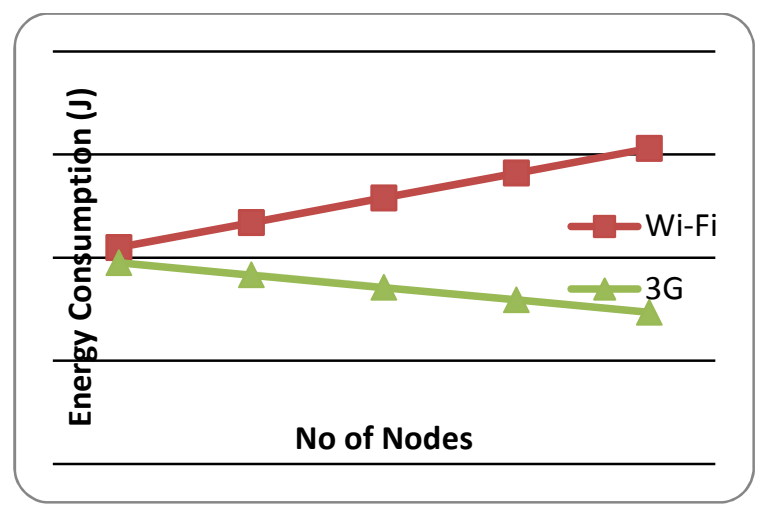

Fig. 16. Comparison of energy consumption between $\mathrm{W} i$ Fi and $3 G$ 
S. Venkatasubramanian et al. / International Journal of Computer Networks and Communications Security, 2 (2), February 2014

In general, energy is consumed during transmission, reception, idle and sleep mode of operation. The energy consumption vs. the number of nodes for the comparison between $\mathrm{Wi}-\mathrm{Fi}$ and Bluetooth is shown in Figure 16. Energy consumption is calculated based on the primary energy and the remaining energy of all nodes associated with the network.

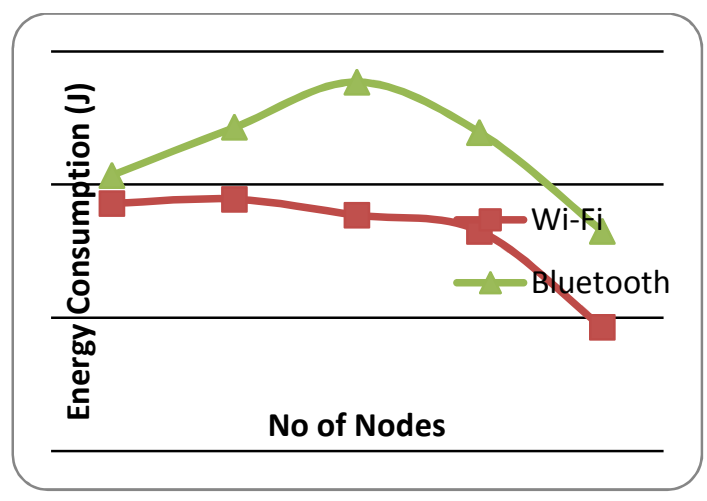

Fig. 17. Comparison of energy consumption between $\mathrm{Wi}$ Fi and Bluetooth

When compared with the Bluetooth, the Wi-Fi consumes less energy than the Bluetooth which is depicted in Figure 17.

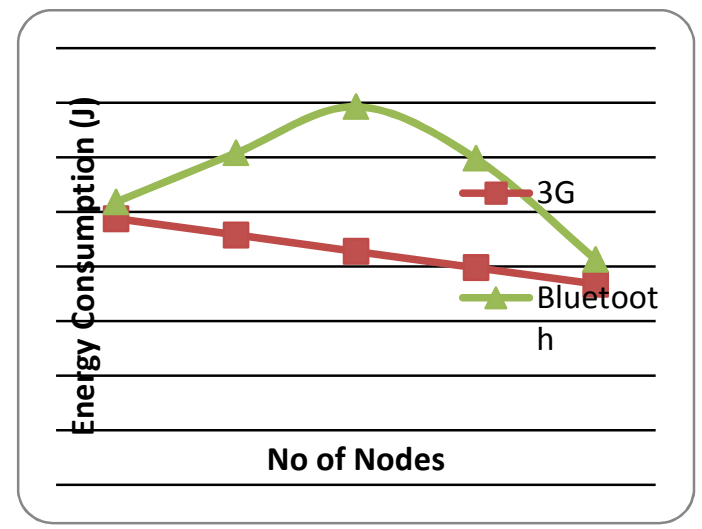

Fig. 18. Comparison of energy consumption between Bluetooth and $3 G$

In Figure 18, we note that the $3 \mathrm{G}$ network has better energy consumption when compared with the Bluetooth network for each set of connections. From these results we conclude that our proposed protocol has better energy consumption when the simulation is carried out and energy consumption increases because of number of packets are received through a selected gateway. As observed from these figures the proposed EEQMRP protocol gives better energy consumption for the $3 \mathrm{G}$ network when compared with the other networks.

\subsubsection{Performance Analysis With Existing Protocol}

\subsubsection{Based On Nodes}

In our experiment the results are based on varying the number of nodes as 20,30, 40 and 50 .

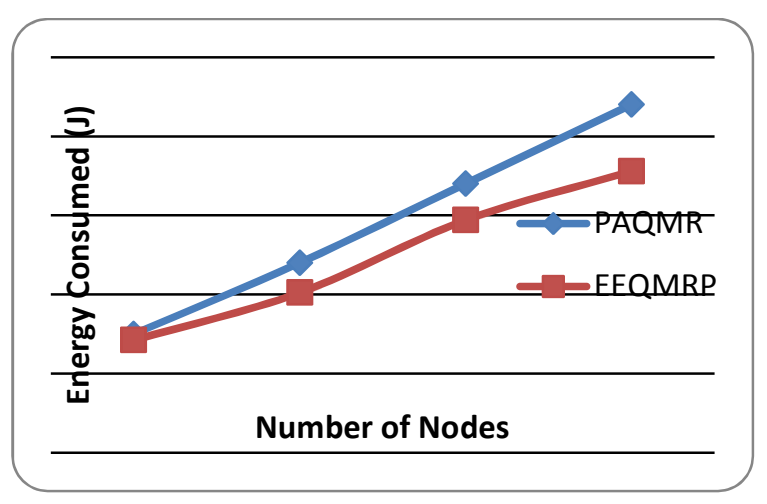

Fig. 19. Nodes Vs Energy Consumption (J)

In figure 19, 20 to 50 nodes network is considered; it can be seen from the figure that in EEQMRP the consumed energy is reduced by including the energy constraint during the path selection when compared to PAMQR.

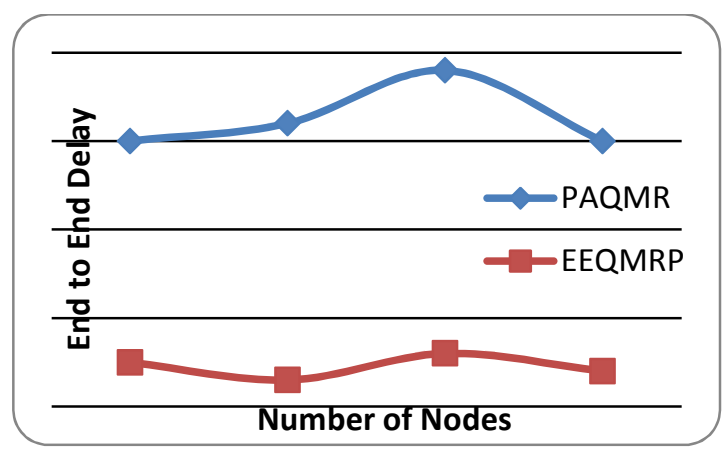

Fig. 20. Nodes Vs End to End Delay

In figure 20, 20 to 50 nodes network is considered; it can be seen from the figure that in EEQMRP the end to end delay is highly reduced while comparing with PAMQR. 


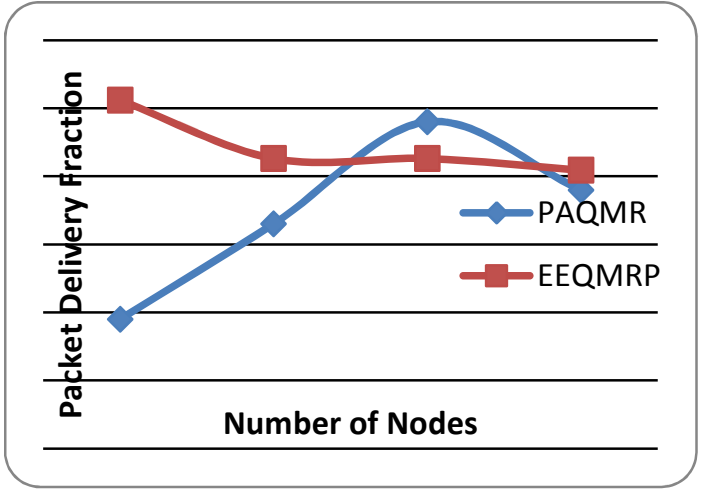

Fig. 21. Nodes VS Packet Delivery Fraction

In figure 21,20 to 50 nodes network is considered; it can be seen from the figure that in EEQMRP the packet delivery fraction increases while comparing with PAMQR.

\section{CONCLUSION}

In MANET networks transmission is possible anytime, which means nodes can connect and disconnect at anytime. Here, we designed the heterogeneous network and multipath routings are constructed by using the QoS specification. Firstly, we recover the multipath routes and then the gateway node is found by using a gateway selection procedure. Then, the multipath for each source to destination is guessed in the network. By taking advantage of the QoS based Energy Efficient Optimal Multipath route discovery and route maintenance in multipath routing protocol, the optimal path for data transmission from source to destination is discovered based on the energy. Using the multipath routing proposed in this paper, the optimal path for data transmission from source to destination is achieved. In this way, QoS parameters such as bandwidth, throughput, packet delivery ratio and end-to-end delay are computed and accomplished with best results.

\section{REFERENCES}

[1] Peng Zhao, Xinyu Yang, Wei Yu, and Xinwen $\mathrm{Fu}$, "A Loose Virtual Clustering based Routing for Power Heterogeneous MANETs", IEEE Transaction accepted paper for future publication, 2011.

[2] Manoharan, Ilavarasan, Rajarajan, "Adaptive Gateway Management in Heterogeneous Wireless Networks", International Journal of Research and Reviews in Ad-Hoc Networks, Vol. 1, No. 2, 2011.
[3] Peter Magula, "QMMAC - New QoS Model with Admission Control for Mobile Ad hoc Networks", 2011.

[4] Pinki Nayak, Rekha Agarwal, Seema Verma, "An Overview of Energy Efficient Routing Protocols in Mobile Ad-Hoc Network", International Journal of Research and Reviews in Ad-hoc Networks, Vol. 2, No. 1, 2012.

[5] S. Uma and S.P. Shantharajah, "Optimization of Power Consumption in Mobile Adhoc Network Communication Systems", Vol 2, Issue 4, ISSN 2319-9725, April 2013.

[6] Soundararajan, Bhuvaneswaran, "Adaptive Multi-Path Routing for Load Balancing in Mobile Ad-Hoc Networks", Journal of Computer Science, Vol. 8, pp. 648-655, 2012.

[7] Floriano De Rango, Peppino Fazio, "LinkStability and Energy Aware Routing Protocol in Distributed Wireless Networks", IEEE Transactions on Parallel and Distributed Systems, Vol. 23, No. 4, pp. 713-726, 2012.

[8] Chandrakant N, Deepa Shenoy P, Venugopal K $R$, "Middleware Services for Security in Scalable and Non-Scalable Heterogeneous Nodes of MANETs", International Journal of Future Generation Communication and Networking, Vol. 4, 2011.

[9] S.Santhi, Dr.G.Sudha Sadasivam, "Power Aware Qos Multipath Routing Protocol For Disaster Recovery Networks", International Journal of Wireless \& Mobile Networks (IJWMN) Vol. 3, No. 6, December 2011.

[10] Seung-Hoon Lee, Starsky H.Y. Wong, Chi-Kin Chau, Kang-Won Lee, Jon Crowcroft and Mario Gerla, "InterMR: Inter-MANET Routing in Heterogeneous MANETs"

[11] R.Hemalatha, M.Yamuna Devi, S.Aishwarya and J.Vijitha Ananthi, "Improving Qos Performance in the Gateway Node", International Journal of Advanced Research in Electrical, Electronics and Instrumentation Engineering, Vol. 2, Issue 2, February 2013.

[12] Venkatasubramanian, Gopalan, "A QoS-Based Robust Multipath Routing Protocol for Mobile Ad-hoc Networks", IACSIT International Journal of Engineering and Technology, Vol.1, No.5, 2009.

[13] Venkatasubramanian, Gopalan, "A Quality of Service Architecture for Resource Provisioning and Rate Control in Mobile Ad-Hoc Networks", International Journal of Ad-hoc, Sensor \& Ubiquitous Computing, Vol.1, No.3, 2010.

[14] Suri, Soni, Parul Tomar, "Cluster Based QoS Routing Protocol for MANET”, International 
Journal of Computer Theory and Engineering, Vol. 2, No. 5, 2010.

[15]R.Vadivel, B.Narasimhan, "Reliable Geographic Routing Protocol (RGRP) towards Improving Quality of Service (QoS) in Heterogeneous Mobile Ad Hoc Networks", International Journal of Computer Applications, Volume 60- No.15, December 2012.

[16]Hung-Chi Chu, Shih-Lung Chang, YingHsiang Liao, and Yan-Ting Pan, "Design and Implementation of Heterogeneous Wireless Gateway”, IEEE, 2009.

[17] Nico Bayer, Dmitry Sivchenko, Bangnan Xu, Sven Hischke, Veselin Rakocevic and Joachim Habermann, "Integration of Heterogeneous Ad hoc Networks with the Internet".

\section{AUTHOR PROFILES:}

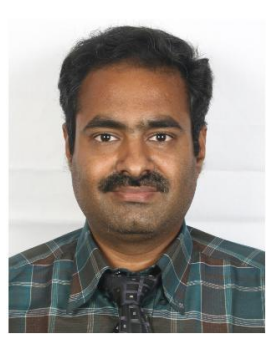

S.Venkatasubramanian received the B.E. degree in Electronics and Communication from Bharathidasan University and M.E. degree in Computer science from Regional Engineering College. He has 16 years of teaching experience. $\mathrm{He}$ is currently pursuing doctoral research in mobile Ad hoc networks. His areas of interest include mobile networks, Network Security and software engineering. At present he is working as Associate Professor in Department of CSE at Saranathan college of Engineering, Trichy, India.

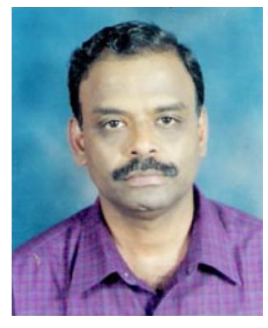

Dr.N.P.Gopalan is a Professor in the Department of computer application at National Institute of Technology, Tiruchirapalli. He received the M.E. degree in Computer science from Regional Engineering College. and Ph.D degree in theoretical computing from Indian Institute of Science .He has more than 30 years of teaching experience. He has published more than papers in International and National journals and conference proceedings. $\mathrm{He}$ has published 3 books. His areas of research include Data mining, Web Technology, Distributed Computing, and Theoretical Computer Science. 